\title{
Highly Emissive Biological Bilirubin Molecules: Shedding New Light on the Phototherapy Scheme
}

\author{
Ahmed M. El-Zohry,* Valentin Diez-Cabanes, Mariachiara Pastore,* Taha Ahmed, and Burkhard Zietz
}

Cite This: J. Phys. Chem. B 2021, 125, 9213-9222

Read Online

ABSTRACT: Bilirubin (BR) is the main end-product of the hemoglobin catabolism. For decades, its photophysics has been mainly discussed in terms of ultrafast deactivation of the excited state in solution, where, indeed, BR shows a very low green emission quantum yield (EQY), $0.03 \%$, resulting from an efficient nonradiative isomerization process. Herein, we present, for the first time, unique and exceptional photophysical properties of solidstate $\mathrm{BR}$, which amend by changing the type of crystal, from a closely packed $\alpha$ crystal to an amorphous loosely packed $\beta$ crystal. BR $\alpha$ crystals show a very bright red emission with an EQY of ca. $24 \%$, whereas $\beta$ crystals present, in addition, a low green EQY of ca. $0.5 \%$. By combining density functional theory (DFT)
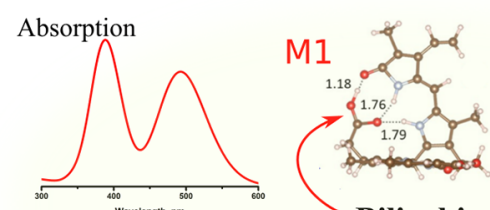

Emission
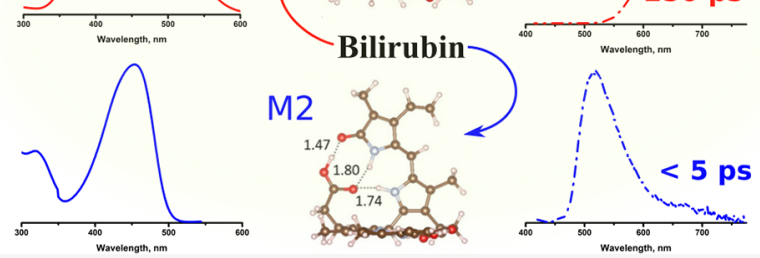
calculations and time-resolved emission spectroscopy, we trace back this dual emission to the presence of two types of BR molecules in the crystal: a "stiff" monomer, M1, distorted by particularly strong internal H-bonds and a "floppy" monomer, M2, having a structure close to that of BR in solution. We assign the red strong emission of BR crystals to M1 present in both the $\alpha$ and $\beta$ crystals, while the low green emission, only present in the amorphous $(\beta)$ crystal, is interpreted as M2 emission. Efficient energy-transfer processes from M2 to M1 in the closely packed $\alpha$ crystal are invoked to explain the absence of the green component in its emission spectrum. Interestingly, these unique photophysical properties of BR remain in polar solvents such as water. Based on these unprecedented findings, we propose a new model for the phototherapy scheme of BR inside the human body and highlight the usefulness of BR as a strong biological fluorescent probe.

\section{INTRODUCTION}

Bilirubin IX $\alpha$ (termed BR hereafter) is among important biological metabolites which undergoes biologically relevant processes through interaction with light, such as rhodopsin (causing vision) $)^{1,2}$ and chlorophyll (photosynthesis in plants). ${ }^{3}$ $\mathrm{BR}$ is formed mainly from the degradation cycle of hemoglobin, and it is naturally excreted with the help of liver, after binding to human serum albumin (HSA) and other sugar groups. ${ }^{4-6}$ In newborns, however, the liver might not be active for few days after delivery, causing an accumulation of neurotoxic BR, which manifests itself as neonatal jaundice, affecting the brain functionality and leading to death in extreme cases. There is, thus, an urgent necessity to remove the accumulated BR. This is commonly performed by resorting to BR phototherapy, that is, by exposing the patient to irradiation in the visible range. ${ }^{7}$ The current established mechanism for $\mathrm{BR}$ excretion is that blue-to-green light converts the insoluble $\mathrm{BR}$ isomers, through the isomerization process, into soluble ones that can be excreted. ${ }^{8} \mathrm{BR}$ is a highly nonpolar molecule that tends to interact with lipids rather than with physiological water molecules. ${ }^{9}$ Despite several studies about the photodynamics of $\mathrm{BR}$, many processes are not well understood yet because of the complexity of the BR chemical structure. ${ }^{10-14}$
Because of the insolubility of BR in most solvents, the photophysical properties of BR in vitro, or upon interaction with HAS, have been studied mainly in nonpolar or basic media to prepare clear solutions of $\mathrm{BR}$, that is, the soluble form of BR. ${ }^{15-18}$ These photophysical studies indicate that, upon photoexcitation, an efficient ultrafast isomerization takes place, converting the Z,Z-BR isomer into a mixture of $\mathrm{Z}, \mathrm{E}$ isomers with a reaction quantum yield of $10 \%$, while the rest retains back to the Z,Z-BR isomer. ${ }^{10,12,19}$ In $\mathrm{CHCl}_{3}$, BR shows a very weak emission quantum yield (EQY) of $0.03 \%\left(\tau_{\mathrm{obs}} \sim 0.3 \mathrm{ps}\right.$, $\tau_{\mathrm{r}} \sim 1.0 \mathrm{~ns}$ ), which increases to $0.3 \%$ upon binding to HAS that restricts the dominating isomerization process of soluble BR. $^{12,20}$

Despite the importance of the previous studies, we think this is not the best way to obtain biologically relevant insights into the BR photophysics because it is not really connected to the

Received: June 16, 2021

Revised: July 17, 2021

Published: August 4, 2021 
expected behavior of BR molecules in the human body or mammals in general, in which water is the main physiological medium. Simultaneously, because of the inactivity of the liver in the early days of the newborns, BR is expected to be present as small solid particles or as suspensions in the human body, especially if not bound to the HSA. This highlights the importance of studying BR in the solid form or as suspensions in proper solvents, such as water, to fully understand the reaction pathways of suspended BR particles before excretion under physiological conditions. Unfortunately, to date, there is no such photophysical study on the BR in the solid state.

Herein, we report, for the first time, a photophysical study on the $\mathrm{BR}$ in the solid state, by combining first principles calculations and time-resolved emission spectroscopy. We reveal unique strong emission properties of the BR crystals, and we rationalize these peculiar features on the base of the crystal structure. These unprecedented findings have been extended as well to suspensions in water. Understanding the behavior of $\mathrm{BR}$ in the solid state should provide information on the behavior of BR particles in the newborns before excretion, as well as on the overall picture of the excited-state dynamics of BR molecules inside human bodies under phototherapy procedure.

\section{METHODS}

Chemicals. The solvents, acetonitrile and $\mathrm{CHCl}_{3}$ (both Sigma-Aldrich, spectrophotometric grade), $\mathrm{MeOH}$ (SigmaAldrich, Chromasolve), and distilled water were used without further purification. BR was purchased from Sigma-Aldrich as a mixed isomer sample and from Frontier Scientific Porphyrin Products.

Steady-State Spectroscopy. Absorption spectra were measured on a Varian Cary 5000, and emission measurements were performed using a Horiba Jobin Yvon Fluorolog and automatically corrected for wavelength-dependent instrument sensitivity. The reflectance measurements have been measured for solid samples as well to accommodate for the expected scattering effect. For the sake of clarity, the absorption data are shown herein as there were no significant differences between transmission and reflectance data. Solution measurements were carried out at the right angle in a $1 \mathrm{~cm}$ cuvette, while glass films and tapes were measured using the front-face geometry (ca. $30^{\circ}$ ). In order to remove self-absorption effects, the peak optical densities were maintained below 0.1 at the excitation wavelength. The front-face position was used for samples on glass slides. Emission spectra were recorded at $400 \mathrm{~nm}$ excitation wavelength. To measure the emission from the solid particles, $\mathrm{BR}$ was dissolved in $\mathrm{CHCl}_{3}$ and then spread over a glass plate until the end of solvent evaporation (crystals $\beta$ ). In this procedure, small and fuzzy particles were highly expected to be formed. Also, BR solid particles were directly used from the used batch on a plastic tape by taking background measurements to detect the emission crystals $\alpha$. Upon adding organic bases to the suspensions of BR crystals in solutions, small volumes of concentrated bases were utilized and added directly to the suspension solutions without prior dilution. Thus, the presented data upon adding organic bases show qualitative data than quantitative ones.

Time-Resolved Measurements: Time-Correlated Singel Photon Counting (TCSPC) and Streak Camera. The streak camera setup has been described in detail previously. ${ }^{21}$ Briefly, the excitation of the sample with ultrafast laser pulses was performed using a frequency-doubled Ti:Sa oscillator
(Coherent Mira) output $(400 \mathrm{~nm})$ at $76 \mathrm{MHz}$. A $1.0 \mathrm{~cm}$ cuvette was used (for suspensions), and the laser beam was directed into the cuvette close to the cuvette wall on the emission side, thus reducing the efficient cuvette length to 1-2 $\mathrm{mm}$. Fluorescence at a right angle to the excitation was passed through a Bruker SPEC 250IS and onto the streak camera. The charge-coupled device (CCD) camera was used in the binning mode $(2 \times 2$ pixels $)$ to give a $512 \times 512$ pixel matrix. The global fit procedure has been used for the kinetic analysis, and all the obtained data are presented in Table 1. Also, amplitudes

Table 1. Extracted Emission Lifetimes of BR under Various Conditions Using the Global Fitting Procedure

$\begin{array}{lcc}\text { environment } & @ 550 \mathrm{~nm} / \mathrm{ps} & @ 700 \mathrm{~nm} / \mathrm{ps} \\ \mathrm{MeCN} & 4.3 \pm 0.1 & 260 \pm 4 \\ \mathrm{MeOH} & 3.1 \pm 0.2 & 250 \pm 15 \\ \text { water } & 5 \pm 1.1 & 265 \pm 5 \\ \boldsymbol{\alpha} \text { crystals } & 25 \pm 2.8 & 240 \pm 10 \\ \boldsymbol{\beta} \text { crystals } & 5 \pm 1 & 140 \pm 6\end{array}$

are calculated from the absolute integral area under the decayassociated spectra. Herein, the Instrumental Response Function (IRF) of the utilized pulse is about 5 ps.

Scanning Electron Microscopy and Powder X-Ray Diffraction Techniques. The morphology of the BR powders was characterized by field emission scanning electron microscopy (FE-SEM) (LEO 1550, Schottky FEG, ZrO/W cathode) in the secondary electron detection mode at an accelerating voltage of $300 \mathrm{~V}$, an aperture size of $30 \mu \mathrm{m}$, zero tilt, and averaging 100 frames. The sample (powder) was spread thin on a regular conductive carbon tape-covered aluminum stub. The powders were imaged as is without sputtering any conductive layer on top. The sample (powder) was suspended in $\mathrm{EtOH}$; the slurry was homogenized briefly with a mortar and pestle, spread on a silicon sample holder, and allowed to dry at ambient conditions (shielded from strong light sources). Crystal structure characterizations were performed by powder X-ray diffraction (PXRD), with a Bruker D8 diffractometer using CuK $\alpha 1$ radiation, $\lambda=1.5406 \AA$, in the Bragg-Brentano configuration equipped with a LYNXEYE linear detector. The measurements were made using a $2 \theta$ range of $2-40^{\circ}$, with a rotating sample holder.

Computational Details. All theoretical calculations were conducted at the density functional theory (DFT) level within the wB97XD functional ${ }^{22}$ and $6-31 G(d)$ as the basis set. The choice of this functional has been motivated because of its capability to properly describe charge-transfer (CT) states by means of long-ranged correlations and dispersion effects via Grimme D2 empirical correction. The geometries of the monomers and aggregates (pentamers) conforming the crystal structure were extracted from the available X-ray diffraction (XRD) data. ${ }^{23}$ For these systems, the calculations were performed in the gas phase, whereas for the BR molecules in solution, the solvent environment was accounted for by the conductive polarizable continuum medium (CPCM) method. ${ }^{24}$ The excited-state properties were estimated by means of time-dependent DFT (TD-DFT) formalism and following the same level of theory as the one used for the ground state. The assignment of the exciton localization along the two fragments conforming the BR molecule (see Figure S1) has been performed by employing the transition density matrix manipulation tools implemented in TheoDORE package. ${ }^{25}$ 


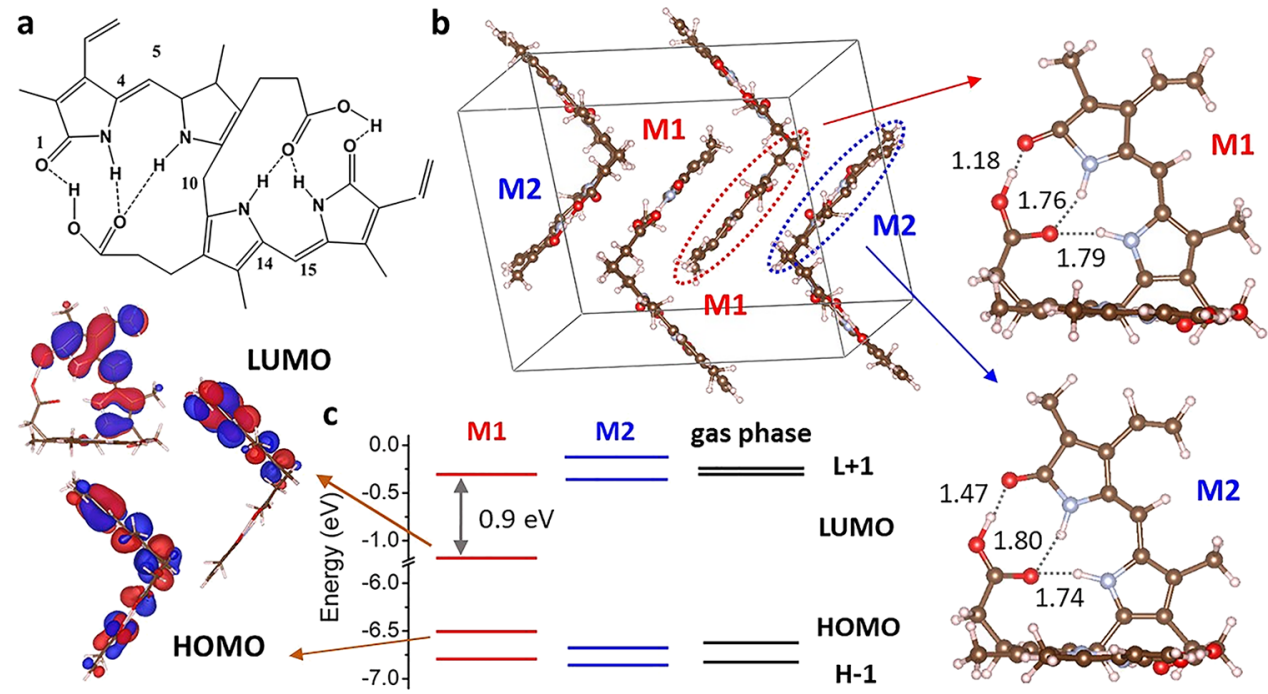

Figure 1. (a) Structure of the Z,Z BR isomer, showing the internal hydrogen bonds. (b) Perspective view of the unit cell forming the BR crystals made of two pairs of monomers (M1 and M2), which are displayed on the right part of the image. The H-bond distances highlighted here are given in Å. (c) Frontier energy levels for M1 (red), M2 (blue), and BR in the gas phase (black), with the isodensity plots of the LUMO and HOMO of M1 represented in the left part of the graph. The rest of the frontier molecular orbital isodensity plots are collected in Figure S3. The isovalue used to plot the isodensities was 0.02 a.u.

One the same foot, natural transition orbitals (NTOs) were used to obtain a spatial representation of the lowest energy excitations. ${ }^{26}$ All sets of calculations were performed with the Gaussian 16 package. $^{27}$

Estimation of the Förster Resonance Energy-Transfer (FRET) Properties. The dependency of the relative position of the neighboring monomers M2 and M1 (see Figure S5) has been accounted for by means of the orientation factor $\kappa$, which is calculated as follows

$$
\kappa=\hat{\mu}_{\mathrm{D}} \cdot \hat{\mu}_{\mathrm{A}}-3\left(\hat{\mu}_{\mathrm{D}} \cdot \hat{\mathrm{R}}\right)\left(\hat{\mu}_{\mathrm{A}} \cdot \hat{\mathrm{R}}\right)
$$

where $\mu_{\mathrm{D} / \mathrm{A}}$ represents the normalized transition dipole moment of the donor/acceptor (M2/M1) monomers, and $R$ is the normalized vector connecting the centers of mass for both monomers. Once we calculate the orientation factor $\kappa$, we can determine the Förster distance of this pair, the donor/ acceptor pair, by following eq 2

$$
\begin{aligned}
& R_{0}{ }^{6}=\frac{2.07}{128 \pi^{5} N_{\mathrm{A}}} \frac{\kappa^{2} Q_{\mathrm{D}}}{n^{4}} J(\lambda) \\
& J(\lambda) \equiv \int_{0}^{\infty} F_{\mathrm{D}}(\lambda) \varepsilon_{\mathrm{A}}(\lambda) \lambda^{4} \mathrm{~d} \lambda
\end{aligned}
$$

where $N_{\mathrm{A}}$ is the Avogadro number, $Q_{D}$ is the fluorescent quantum yield of the donor (M2), which is equal to $3 \times 10^{-4}$ according to the experimental measurement, $n$ is the reflective index of the medium set to 1.335 , and $J(\lambda)$ is the spectral overlap integral between the normalized donor M2 emission spectrum and the acceptor molar extinction coefficient $\left(\varepsilon_{\mathrm{A}}\right)_{\max }$ $=60,000 \mathrm{M}^{-1} \mathrm{~cm}^{-1}$, as obtained from the M1 absorption spectrum. These parameters lead to a $J(\lambda)$ value equal to 2.05 $\times 10^{15} \mathrm{M}^{-1} \mathrm{~cm}^{-1} \mathrm{~nm}^{4}$.

\section{RESULTS AND DISCUSSION}

Figure 1a shows the chemical structure of BR, consisting of two almost equivalent units, that is, the endo and exo forms of a dipyrrinone chromophore, linked by a saturated $\mathrm{CH}_{2}$ group. The two halves also form strong internal hydrogen bonds $(\mathrm{H}-$ bonds) that can be broken upon illumination through a fast isomerization process, making the isomerized $\mathrm{BR}$ vulnerable to interact with water and special proteins to be excreted easily through the bile. ${ }^{19}$ The most stable isomer form of BR is called $\mathrm{Z}, \mathrm{Z}-\mathrm{BR}$, which is insoluble in most solvents, including water, because of the strong internal hydrogen bonds. ${ }^{28}$

The crystal structure package, based on the previous XRD results of BR crystals, ${ }^{23}$ shows that two different BR units are present, hereafter indicated as M1 and M2 (see Figures $1 \mathrm{~b}$ and S1). Although the BR monomers may appear quite similar, one fragment of $\mathrm{M} 1$ shows an internal strong $\mathrm{H}$-bond between the $\mathrm{O}$ atoms $(1.18 \AA$ ), which is absent in the M2 fragments (see the right part of Figure $1 \mathrm{~b}$ ). This $\mathrm{H}$-bond is very strong, close to be a real covalent bond, with a distance of $1.18 \AA$ (see also Figure S1 for other fragments). This strong H-bond is also expected to make M1 monomers more restricted and less flexible toward any excited-state large-scale motion and more insoluble in various solvents with respect to M2. Interestingly, DFT calculations performed on the X-ray structures of M1 and M2 disclose that the two highest occupied and lowest unoccupied molecular orbitals (HOMO $-1 / \mathrm{HOMO}$ and LUMO/LUMO + 1) are not-longer quasi-degenerate for M1 (see Figure 1c). Indeed, the structural distortion engenders a sizable stabilization $(\sim 0.9 \mathrm{eV})$ of the LUMO level, being localized on the distorted moiety (see left part Figure 1c), and a slight destabilization of the HOMO level. Consequently, the calculated absorption spectrum (see Figure $2 \mathrm{a}$ and Table S2) presents two distinct absorption peaks, centered around 390 and $490 \mathrm{~nm}$, corresponding to local fragment excitation from the HOMOs to LUMO $+1\left(S_{2}\right)$ and to LUMO $\left(S_{1}\right)$, respectively (see Figure $S 3-4$ ). On the other hand, a single absorption band is calculated for M2, around $385 \mathrm{~nm}$, because of the near-energy degeneracy of the LUMO and LUMO + 1 (Figure 1c). Thus, the absorption spectrum of the crystal package of BR is expected to be a combination of M1 and M2 spectral bands. Note that the absorption features of the monomers are not substantially modified upon interaction with the neighboring molecules of the crystal (see Table S3). We 
a.
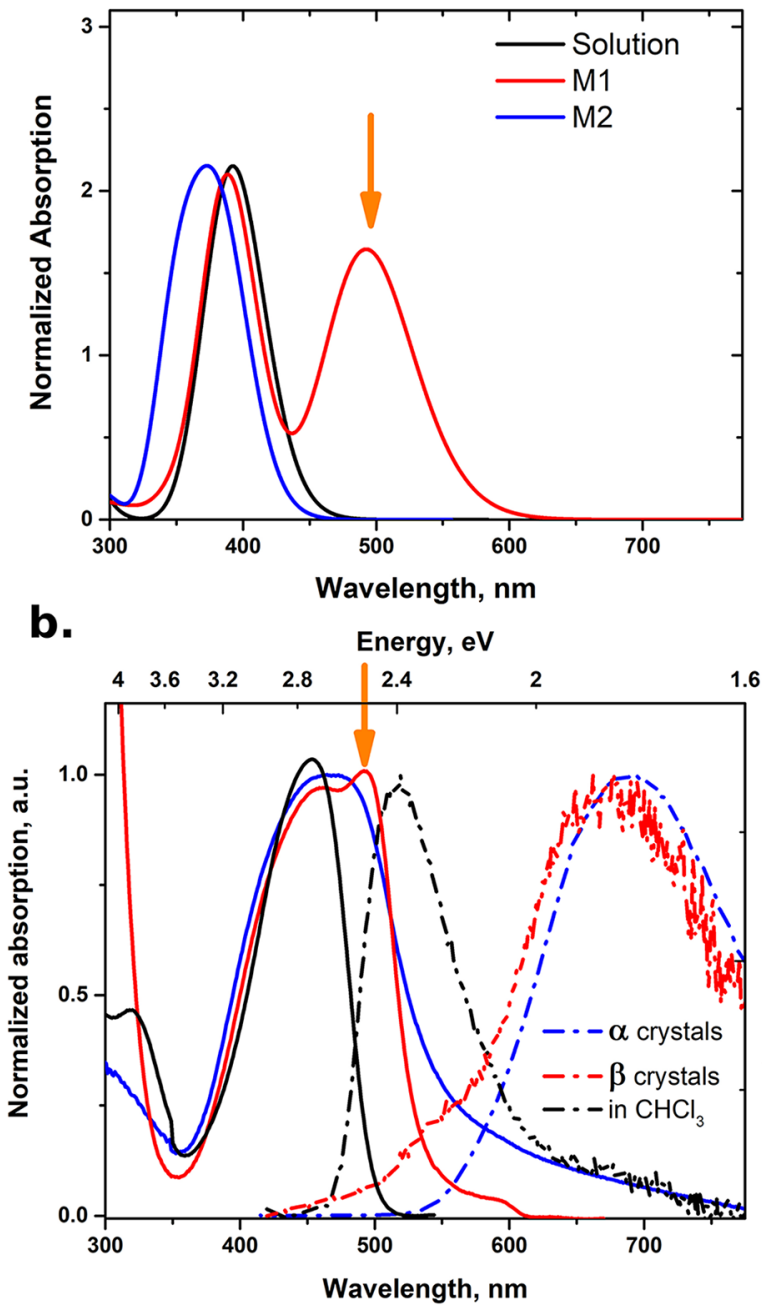

C.

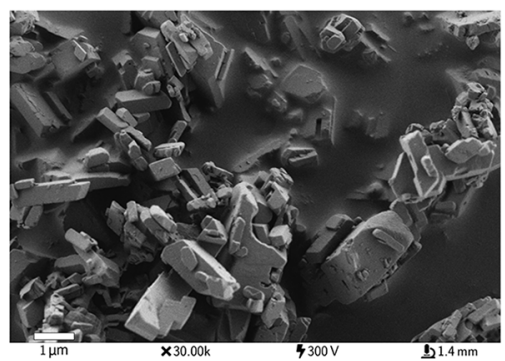

d.

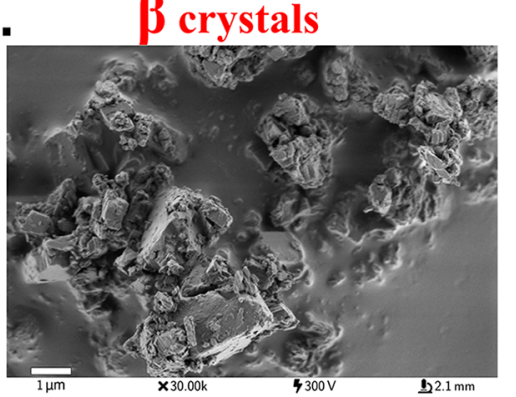

e.

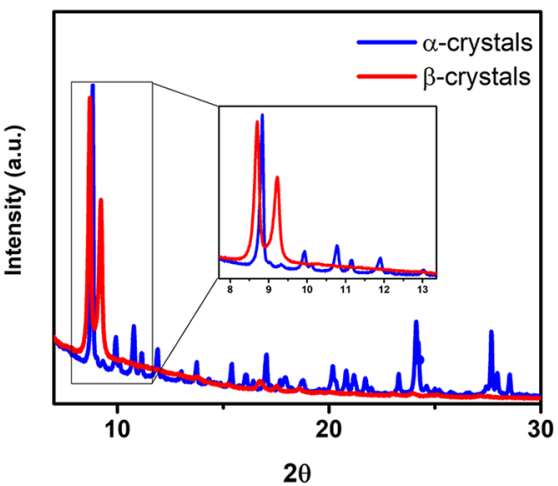

Figure 2. (a) Calculated absorption spectra of M1 and M2. The nature of the main transitions is reported in Table S2. (b) Experimental absorption and emission of BR in solution and solid state. SEM images for $\alpha$ crystals, M1, M2 (c), and $\beta$ crystals mainly M1 (d). (e) XRD measurements for $\alpha$ and $\beta$ crystals of BR. The XRD data show many isomers in the crystal package. Most of "M2" isomers have been disappeared after rough recrystallizations because of their solubility or their conversion into another isomer "check the new intense peak close to the potential M1 peak."

called this crystal package " $\alpha$ crystals." Interestingly, the simulated spectrum of $\mathrm{BR}$ in $\mathrm{CHCl}_{3}$, after full structural relaxation, exhibits a single absorption peak around $395 \mathrm{~nm}$, which is very close to the calculated maximum absorption of M2 (see Figures 1c and 2a), as expected on the basis of their similar geometrical and electronic properties (see Section 1.1 in the Supporting Information). This indicates that M1 can only be identified in the solid-state BR, where these strong $\mathrm{H}$ bonds can be formed thanks to the crystal packing.

To verify these theoretical observations, absorption and emission of BR $\alpha$ crystals have been studied in crystalline solid form and after dissolution in $\mathrm{CHCl}_{3}$ (see Figure $2 \mathrm{~b}$ ). Upon dissolving the $\mathrm{BR}$ crystals in $\mathrm{CHCl}_{3}$, it has been noticed that the solution is not fully clear and a simple filtration step is needed to obtain a clear solution because of the insolubility of M1. The filtered solution in $\mathrm{CHCl}_{3}$ shows a strong absorption band centered at ca. $450 \mathrm{~nm}$, with a corresponding weak emission band at $520 \mathrm{~nm}$, matching with the previous measurements of $\mathrm{BR}$ in $\mathrm{CHCl}_{3}{ }^{10-12,14}$ However, the BR $\alpha$ crystals (see the SEM image for large crystals, Figure 2c) show a broader absorption band centered at ca. $475 \mathrm{~nm}$, with a significant absorption tail extending to $750 \mathrm{~nm}$ (blue solid and dashed lines in Figure 2b). Interestingly, the absorption band of $\alpha$ crystals matches quite well with that of soluble $\mathrm{BR}$ in $\mathrm{CHCl}_{3}$ (full and dashed black lines in Figure $2 \mathrm{~b}$ ) in the highenergy side of the spectrum. Surprisingly, the emission of $\alpha$ crystals shows a stronger red-shifted band centered at ca. 700 $\mathrm{nm}$. To the best of our knowledge, this observation has not been detected previously for the BR molecules. However, based on our quantum chemical calculations, we can interpret these spectral differences between $\mathrm{BR}$ in $\mathrm{CHCl}_{3}$ and $\mathrm{BR}$ in the solid state as arising from the contribution of M1 monomers. Indeed, while in the BR $\alpha$ crystals, both M1 and M2 forms are present, and the filtered solution may not contain M1 because of its lower solubility caused by the strong intramolecular $\mathrm{H}$ bond mentioned above. To indirectly verify this assumption, we dissolved $\mathrm{BR} \alpha$ crystals in $\mathrm{CHCl}_{3}$, removed most of the clear solution, and re-evaporated $\mathrm{CHCl}_{3}$, forming the $\mathrm{BR}$ crystals again. In this way, we expect to have less M2 monomers in the formed crystals because most of them have been removed by dissolution in $\mathrm{CHCl}_{3}$. The $\mathrm{BR}$ crystals have been imaged by SEM before and after this rough recrystallization process (see Figure $2 \mathrm{c}, \mathrm{d}$ ). Clearly, as shown in the SEM images, this recrystallization process is not 
a.

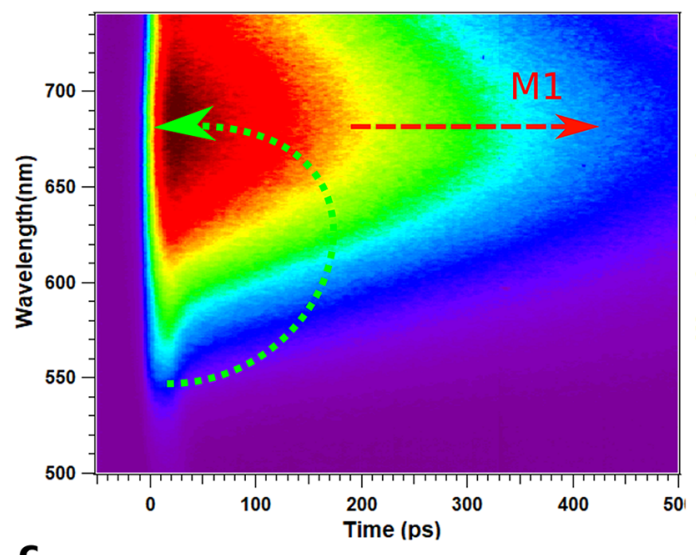

c.

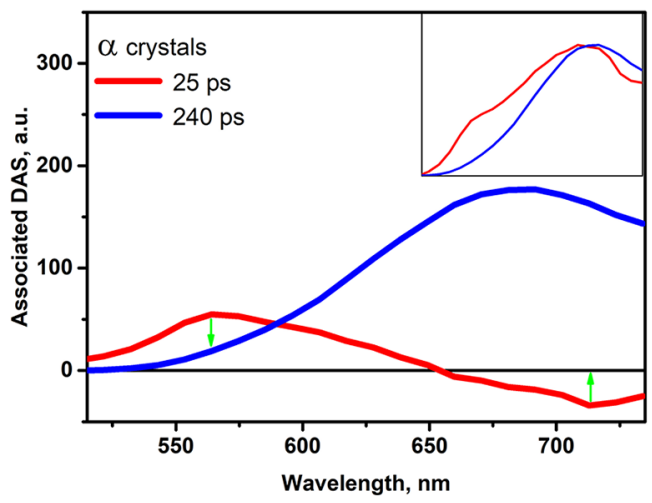

b.

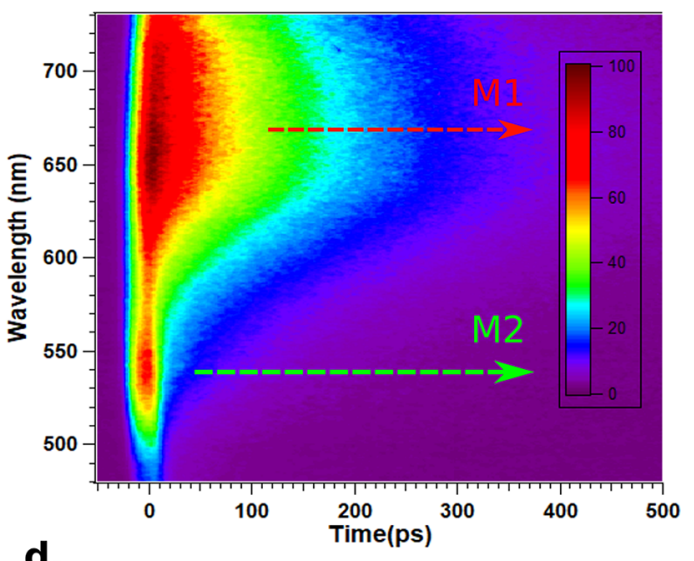

d.

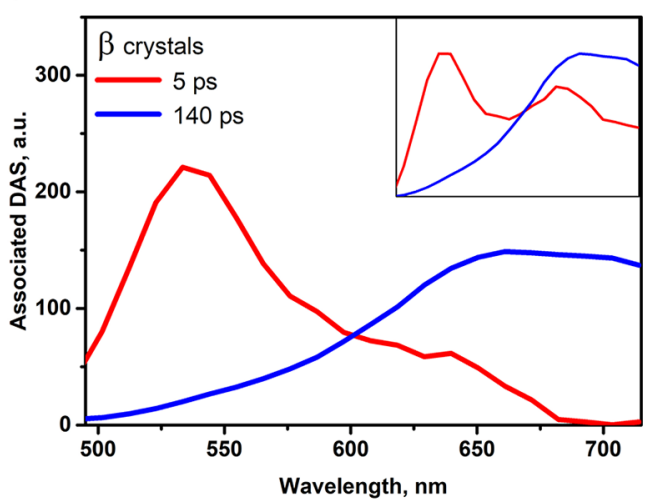

Figure 3. (a) Time-resolved emission properties of BR $\alpha$ crystals. (b) Time-resolved emission of BR $\beta$ crystals. (c) Decay-associated spectra for $\alpha$ crystals for two emission lifetimes. In the inset, the extracted normalized emission spectra at corresponding times $25 \mathrm{ps}$ (red) and $240 \mathrm{ps}$ (blue) are shown. (d) Decay-associated spectra for $\beta$ crystals for two emission lifetimes. In the inset, the extracted normalized emission spectra at corresponding times 5 ps (red) and 140 ps (blue) are shown. See text for more information.

optimum, but still the crystallinity of the BR particles is present, as shown by the XRD measurements (see Figure 2e). These new BR crystals are named " $\beta$ crystals." As expected, the absorption and the emission properties of the two BR crystal forms are different (see the orange arrow in Figure $2 b$ ). The absorption band of the $\beta$ crystals resembles that of the $\alpha$ crystals, but a clear absorption peak at $500 \mathrm{~nm}$ is present. This low-energy absorption peak matches with the calculated lowerenergy absorption band for the M1, which is localized on the "distorted" dipyrrinone unit (see Figure 2a,b). On the other hand, the measured emission of the $\beta$ crystals is slightly different. Although the main emission peak at ca. $700 \mathrm{~nm}$ is still present, the emission is weaker, and there is the appearance of a new emission shoulder at roughly $525 \mathrm{~nm}$. Interestingly, the position of this new emission shoulder almost coincides with the emission band of soluble $\mathrm{BR}$ in $\mathrm{CHCl}_{3}$ (see Figure 2b).

To fully rationalize these peculiar spectral properties, especially the emission ones, we utilized time-resolved emission with picosecond time resolution. The BR $\alpha$ crystals show long-lived emission at room temperature with a corresponding emission lifetime of 240 ps (see Figure 3a). Knowing that the EQY of BR in the solution is about $0.03 \%$ (average emission lifetime is about $0.3 \mathrm{ps}$ ), ${ }^{12,20}$ the EQY for the $\mathrm{BR} \alpha$ crystals is about three orders of magnitude higher ( 24\%). This high EQY of BR $\alpha$ crystals is possibly due to restriction of the ultrafast isomerization process of $B R$, which increase the barrier for the conical intersection in the excited state, as previously shown in similar systems. ${ }^{10-12,29,30}$ Moreover, we interpret this red-emission peak as coming from $M 1$, whose $S_{1}$ state is lower in energy with respect to M2 (492 vs $384 \mathrm{~nm}$ for M1 and M2, respectively). Then, we report transient-emission data measured on the BR $\beta$ crystals in Figure 3b. First, the emission peak at ca. $700 \mathrm{~nm}$ has a shorter lifetime of $140 \mathrm{ps}$, possibly due to the surface defects formed during the recrystallization process. ${ }^{31-33}$ Second, a clear short lifetime emission shoulder at $525 \mathrm{~nm}$ appears, with an average lifetime of 5 ps (EQY 0.5\%) (see Table 1). We assign this short emission peak, which coincides with the emission of BR in solution, to the M2 monomer emission present in the $\beta$ crystals. Interestingly, the EQY of M2 in $\beta$ crystals is similar to that of BR upon binding to HSA, indicating that the isomerization of M2 in $\beta$ crystals is partially restricted. ${ }^{12,20}$

The question that we need to answer now is why the green emission shoulder does not appear in the well-packed $\alpha$ crystals. To validate our assignments and explain the differences in the emission dynamics, we have explored the possibility of realizing Förster resonance energy transfer (FRET) from M2 to M1 among the very close and wellpacked different BR molecules in the $\alpha$ crystals (see details in section 1.6 in the Supporting Information). Herein, invoking a FRET mechanism is not exotic because, previously, FRET was also suggested to occur between the two halves of BR (XBRs) in solutions and then disappear in the XBR molecule, as well as upon deprotonation of BR. ${ }^{10}$ Indeed, FRET rates are 
a.

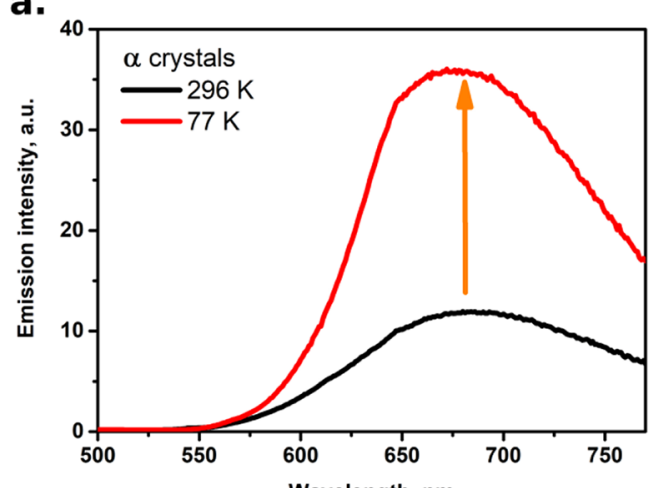

c.

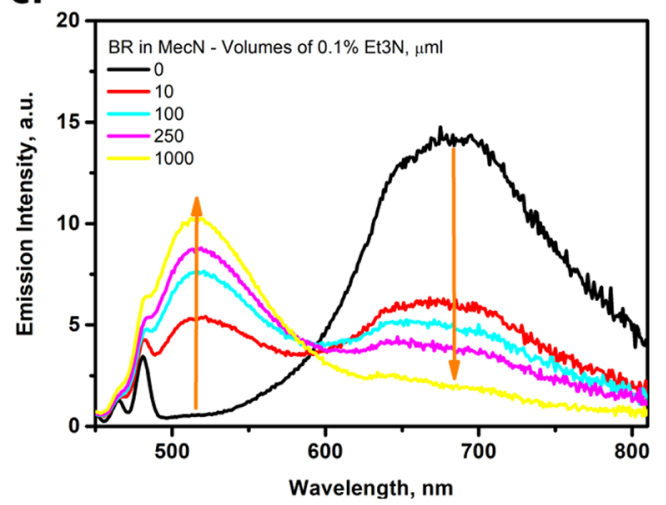

b.

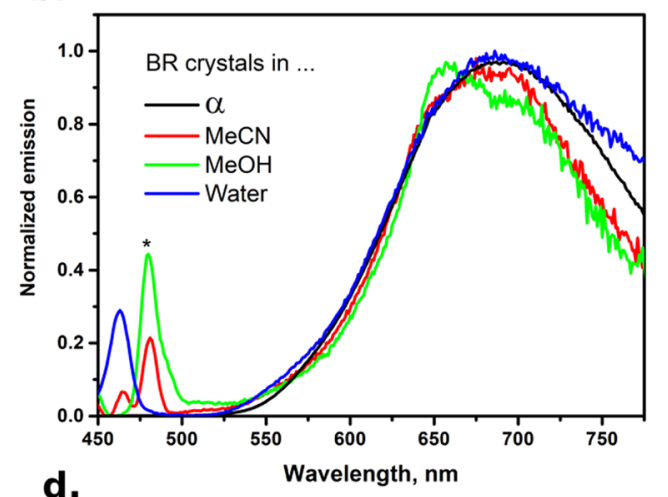

d.

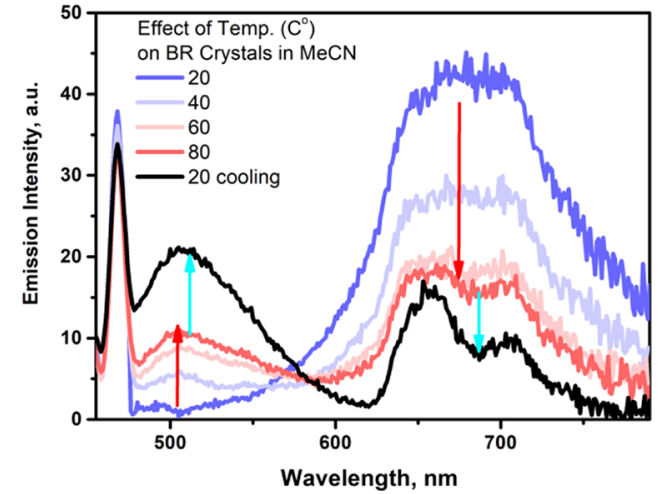

Figure 4. (a) Effect of decreasing the temperature on the emission properties of $\alpha$ crystals. (b) Emission spectra of suspended BR crystals in various solvents, as shown in the legend. The star shows the Raman lines of solvents. (c) Addition of organic base $\mathrm{Et}_{3} \mathrm{~N}$ to the suspension of $\mathrm{BR}$ crystals in $\mathrm{MeCN}$. (d) Effect of heating and cooling the suspensions of BR in MeCN. See text for more information.

dependent on the relative position of the donor-acceptor pairs, which is expressed as follows ${ }^{34}$

$$
k_{\mathrm{F}}=\frac{1}{\tau_{0}} \frac{R_{0}^{6}}{\left|r_{\mathrm{A}}-r_{\mathrm{D}}\right|^{6}},
$$

where $\tau_{0}$ is the lifetime of the donor excited state, $R_{0}$ is the Förster radius, $r_{\mathrm{D}}$ and $r_{\mathrm{A}}$ are the position vectors of the donor (M2) and the acceptor (M1), respectively. The Förster radius, defined as the distance between the donor and the acceptor when the FRET has 50\% probability, can be calculated from the donor luminescence efficiency, the overlap integral of the donor emission spectrum and the acceptor absorption spectrum, and the dimensionless orientation factor $\kappa$, which can vary from 0 to 4 (see the Methods section for more details). The calculated $\kappa^{2}$ values for the four possible combinations of neighboring $\mathrm{M} 2-\mathrm{M} 1$ pairs present in the crystalline structure (see Figure S5 and Table S4) amount to $0.07,0.86,0.78$, and 2.2 . This result manifests an anisotropic transport, with one of the donor-acceptor pair energy transfer being clearly unfavorable with respect to the others. Notably, these values of $\kappa^{2}$ yield to donor/acceptor pair Förster radii values of $10.2,15.6,15.4$, and $18.3 \AA$, being of the same order of magnitude of the FRET radii estimated in common energytransfer processes occurring in biological systems (i.e., $R_{0}$ values for naphthalene-dansy pairs amounted to $23.3 \AA$ ). ${ }^{35}$ Thus, these results corroborate the hypothesis that long-range energy-transfer processes may take place among neighboring M2 and M1 monomers in the $\alpha$ crystal arrangement. The FRET process is also confirmed through the decay-associated spectra (DAS) obtained by fitting the time-resolved data, as shown in Figure 3c,d.
For the $\alpha$ crystals, two emission lifetimes are needed for the global fitting, a short lifetime of 25 ps and a longer lifetime of 240 ps. The DAS for the latter shows an emission-like behavior, as expected. On the other hand, the DAS for the short-lifetime component shows a positive signal around 570 $\mathrm{nm}$ and a negative part centered at $715 \mathrm{~nm}$. This negative part is an indication for the FRET process between the emission components at the blue side (M2) to the ones at the red side (M1) of $\alpha$ crystals through the absorption of M1 monomers. ${ }^{36-38}$ The ratio between the positive and the negative parts of DAS for 25 ps component indicates that the FRET efficiency is at least $60-70 \%$. For the $\beta$ crystals, the DAS of the short component ( $\sim 5 \mathrm{ps})$ shows only an intense positive part extending to $675 \mathrm{~nm}$, indicating the absence of the FRET process between the blue and red-emission sides. Thus, we expect that, upon recrystallization, the ordered crystalline packing is broken, hindering efficient FRET processes between $\mathrm{M} 2$ and $\mathrm{M} 1$ and thus facilitating the appearance of $\mathrm{M} 2$ emission at $\sim 525 \mathrm{~nm}$.

To determine the amount of active nonradiative channels, the emission of $\mathrm{BR}$ crystals has been measured at low temperature $(77 \mathrm{~K})$, and, as it is apparent in Figure $4 \mathrm{a}$, the emission intensity increases by a factor of 3 , meaning that the EQY can reach $\sim 75 \%$. This indicates that nonradiative decay channels are still active at room temperature for the $\alpha$ crystal, suggesting a residual activity of large-scale motions, ${ }^{20,29,30,39-42}$ aggregation effects, ${ }^{21,29,43}$ or heat dissipation by crystal phonons. $^{44,45}$

To correlate the behavior of BR in the crystal form and the one in the physiological environment, we performed emission measurements for suspensions of BR crystals in water and 
Scheme 1. (a) Schematic Representation of the Possible Photophysical Processes in the Solid-State Crystals of BR Crystal versus That in the Solution and (b) Schematic Representation of the Old and the Updated View for the Photoexcited State of BR upon Interaction with the Incident Light in the Human Body

a.

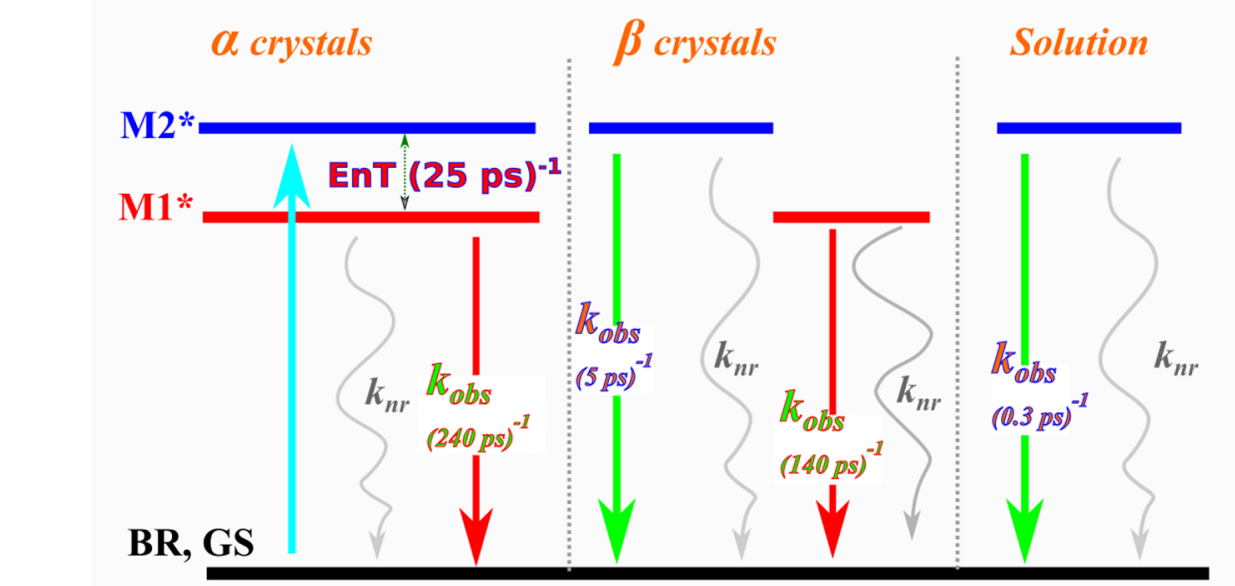

b.

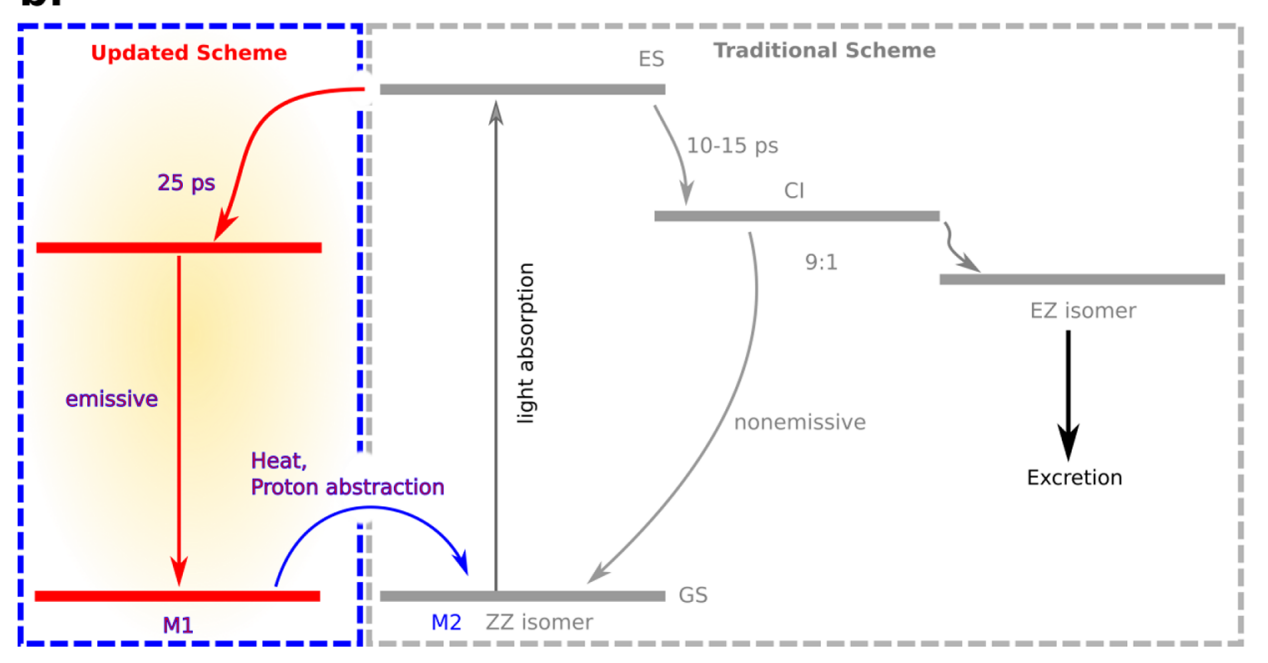

other polar solvents, such as $\mathrm{MeOH}$ and $\mathrm{MeCN}$ (see Figure $4 \mathrm{~b})$. All the emission data show results that are consistent with those measured in the solid phase, indicating that the photophysical properties of BR inside the human body can be rather safely interpreted on the basis of these results. Interestingly, there is also a conversion process between the insoluble M1 and soluble M2 monomers upon changing the basicity of the environment or inducing a thermal effect. Adding small volumes of an organic base (trimethylamine, $\mathrm{Et}_{3} \mathrm{~N}$ ) to the suspensions of $\mathrm{BR}$ in $\mathrm{MeCN}$ shows a systematic suppression of the red-shifted emission and an evolution toward the well-known green emission of BR in the solution. Adding the organic base is expected to deprotonate the BR monomers, breaking the internal strong H-bonds. ${ }^{29,40,46}$ Such an effect of equilibrium between M2 and M1 monomers depends on the solvent as well as on the strength of the employed base, as shown in Figure S6 in the Supporting Information. This is the consequence of the rupture of the strong $\mathrm{H}$-bonds in $\mathrm{M} 1$ and the formation of soluble M2 monomers in the solution. Similarly, by increasing the temperature of the suspended $\mathrm{BR}$ crystals in $\mathrm{MeCN}$, the red emission from M1 monomers decreases, while the green emission of M2 monomers increases. Moreover, this thermal effect is not reversible; thus, cooling the suspensions again to room temperature does not regain the red emission because of the continuous breakdown of the H-bonds and the loss of the symmetry of the crystal packing (see Figure 4d).

Scheme 1a summarizes the current photophysical findings for BR under three different phases. In the well-packed crystals, $\alpha$ crystals, BR is present in the form of M1 and M2 monomers, having different geometries, and electronic properties. Upon light absorption, an efficient FRET process, with a lifetime of $\sim 25$ ps, occurs from M2 to M1, making M1 responsible for the main red-shifted emission at $700 \mathrm{~nm}$ with a QY close to $24 \%$ ( $\tau_{\text {obs }} 240 \mathrm{ps}$ ). For the loosely packed $\beta$ crystals, the energy transfer is hindered and a dual emission, at $\sim 525 \mathrm{~nm}\left(\tau_{\text {obs }} \sim 5\right.$ ps) and at $\sim 700 \mathrm{~nm}\left(\tau_{\mathrm{obs}} \sim 140 \mathrm{ps}\right)$, is measured. When BR is completely soluble, only emission at $525 \mathrm{~nm}$ can be observed, with an EQY of $0.03 \%$ ( $\tau_{\text {obs }} 0.3$ ps $)$ because of the well-known isomerization process. Apparently, the isomerization process and, possibly, other nonradiative deactivation processes are still efficient in the solid state because the BR crystal EQY cannot reach unity even at low temperatures (see Figure 4a). Moreover, in suspensions, by changing the temperature or controlling the environment basicity, one can break the strong intramolecular $\mathrm{H}$-bond characterizing $\mathrm{M} 1$, thus obtaining symmetrical M2 monomers and controlling the emission properties. 
Despite the effectiveness of the phototherapy to treat excess levels of $\mathrm{BR},{ }^{8,47}$ these present findings may shed new light on the current understanding of the phototherapy for the excretion of BR. Previously, no significant efficiency differences have been detected upon using blue light $(\sim 460 \mathrm{~nm})$ or green light $(\sim 500 \mathrm{~nm}) .^{48}$ As mentioned before, all the previous studies on BR focused on the BR in solution, which is on the "M2" monomer in our scheme, and this leads to the picture about the current mechanism, reported in Scheme 1b, for the interaction of M2 with incident light. In this picture, M2 reaches the conical intersection (CI) within $10-15$ ps, and then, isomers of a different ratio 9:1 (ZZ:EZ) are formed. ${ }^{10-12}$ Inserting M1 monomers into the same picture opens another deactivation channel (FRET to M1) for the excited M2 monomers, thus decreasing the efficiency to form excretable EZ isomers. Because M1 monomers have strong internal $\mathrm{H}$ bonds and are highly hydrophobic, absorption of light by M1 monomers is expected to be useless rather than the expected red emission, which might provide the jaundice color for infants. As we have shown, M1 can be converted into excretable M2 monomers by heating because of the incident light or proton abstraction by some proteins. However, these are expected to be slow processes, $\sim$ hours to days, thus requiring prolonged exposure to irradiation, with the correlated higher risks for newborns' health, such as risk of skin cancer, as it is the case in the current phototherapy protocol. ${ }^{8,49}$ However, further investigations in vivo are needed to confirm this hypothesis.

\section{CONCLUSIONS}

Despite the biological relevance of $\mathrm{BR}$, and the understandable great interest devoted to characterize its photophysics in the past years, previous studies have only addressed its absorption and emission properties in solution, which, however, does not mimic the actual biological environment, where BR is likely to be present as small solid particles or as suspensions when not bound to the HAS. Here, for the first time, we have shown that BR single crystals present a unique and completely different photophysical behavior with respect to that reported in the literature. The analysis of the BR crystal ( $\alpha$ crystals) structure and quantum chemical calculations discloses those in the solidstate phase; $\mathrm{BR}$ is present in two different monomeric units, namely, M1 and M2. Particularly, strong internal hydrogen bonds make M1 very poorly soluble and slightly distorted in the crystal packing with respect to $\mathrm{M} 2$ and to the BR structure in the solution. This structural distortion leads to a sizable stabilization of the lowest unoccupied energy levels in M1 and, thus, to a marked shift at longer wavelengths of both absorption and emission, occurring at ca. $700 \mathrm{~nm}$ with a high EQY of ca. $24 \%$. When dissolving BR $\alpha$ crystals, the more soluble M2 molecules are dissolved, and after the irradiation of the filtered solution, the well-known and efficient isomerization process decreases the EQY to $0.03 \%$. We also obtained different BR crystals ( $\beta$ crystals), mainly composed of the lesssoluble M1 units, after the dissolution of $\alpha$ crystals in $\mathrm{CHCl}_{3}$, elimination of most of the clear solution, and solvent reevaporation. The different crystal forms have strikingly different photophysics, that we have shown, based on theoretical calculations, originate from the possibility of realizing efficient long-range energy-transfer (FRET) processes from $\mathrm{M} 2$ to $\mathrm{M} 1$ in the original $\alpha$ crystals, solely leading to the emission at $700 \mathrm{~nm}$. Inefficient FRET in $\beta$ crystals gives rise to dual emission, at ca. 525 and $700 \mathrm{~nm}$, from M2 and M1, respectively. Similar photophysical properties have been detected for BR particles in water as a physiological environment, suggesting that the observed photophysical properties in the solid state can also occur under the phototherapy procedure. Thus, we suggest a more complex phototherapy scheme of $\mathrm{BR}$, which may explain the prolonged exposition to light irradiation that is required for an effective treatment. Therefore, our study opens a new dimension for understanding the BR excited-state dynamics for an efficient phototherapy.

\section{ASSOCIATED CONTENT}

SI Supporting Information

The Supporting Information is available free of charge at https://pubs.acs.org/doi/10.1021/acs.jpcb.1c05308.

Lateral views of the two fragments $\mathrm{A}$ and $\mathrm{B}$ conforming the $\mathrm{BR}$ molecules; lateral and perspective views of the superimposed M1 (red) vs M2 (blue), M1 vs ground state (GS), and M2 vs GS (black) structures; root-meansquare-displacement values of the internal coordinates; frontier molecular orbital shapes for M1, M2, and BR optimized in the gas phase; excitonic properties for the lowest-energy transitions bands of the simulated spectra; NTOs for the two lowest energy excitations of M1, M2, and $\mathrm{BR}$ molecules in the $\mathrm{CHCl}_{3}$ solution; top views of the four different nearest neighbor pentamers; excitonic properties for the lowest-energy transitions of the monomer (gray) and pentamer (yellow) conformations; and orientation factors $\left(\kappa^{2}\right)$ and Förster distances $\left(R_{0}\right)$ for the donor/acceptor pairs (PDF)

\section{AUTHOR INFORMATION}

\section{Corresponding Authors}

Ahmed M. El-Zohry - Department of Chemistry, Ångström Laboratories, SE-75120 Uppsala, Sweden; Department of Physics - AlbaNova Universitetscentrum, Stockholm University, SE-10691 Stockholm, Sweden; 이이.org/ 0000-0003-2901-5815; Email: ahmed.elzohry@fysik.su.se, amfzohry@yahoo.com

Mariachiara Pastore - Université de Lorraine \& CNRS, Laboratoire de Physique et Chimie Théoriques (LPCT), F54000 Nancy, France; 이이. orcid.org/0000-0003-4793-1964; Email: mariachiara.pastore@univ-lorraine.fr

\section{Authors}

Valentin Diez-Cabanes - Université de Lorraine \& CNRS, Laboratoire de Physique et Chimie Théoriques (LPCT), F54000 Nancy, France

Taha Ahmed - Department of Chemistry, Angström Laboratories, SE-75120 Uppsala, Sweden; O orcid.org/ 0000-0001-6717-0408

Burkhard Zietz - Department of Chemistry, Ångström Laboratories, SE-75120 Uppsala, Sweden

Complete contact information is available at: https://pubs.acs.org/10.1021/acs.jpcb.1c05308

\section{Notes}

The authors declare no competing financial interest.

\section{REFERENCES}

(1) Polli, D.; Altoe, P.; Weingart, O.; Spillane, K. M.; Manzoni, C.; Brida, D.; Tomasello, G.; Orlandi, G.; Kukura, P.; Mathies, R. A.; 
et al. Conical Intersection Dynamics of the Primary Photoisomerization Event in Vision. Nature 2010, 467, 440-443.

(2) Broser, M.; Spreen, A.; Konold, P. E.; Peter, E.; Adam, S.; Borin, V.; Schapiro, I.; Seifert, R.; Kennis, J. T. M.; Bernal Sierra, Y. A.; et al. Neor, a near-Infrared Absorbing Rhodopsin. Nat. Commun. 2020, 11, 5682.

(3) Kato, K.; Shinoda, T.; Nagao, R.; Akimoto, S.; Suzuki, T.; Dohmae, N.; Chen, M.; Allakhverdiev, S. I.; Shen, J. R.; Akita, F.; et al. Structural Basis for the Adaptation and Function of Chlorophyll $\mathrm{F}$ in Photosystem I. Nat. Commun. 2020, 11, 238.

(4) Dosch, A. R.; Imagawa, D. K.; Jutric, Z. Bile Metabolism and Lithogenesis: An Update. Surg. Clin. North Am. 2019, 99, 215-220.

(5) McDonagh, A. F.; Palma, L. A.; Lightner, D. A. J. S. Blue Light and Bilirubin Excretion. Science 1980, 208, 145-151.

(6) McDonagh, A. F.; Lightner, D. A. Like a Shrivelled Blood Orange-Bilirubin, Jaundice, and Phototherapy. J. Pediatrics 1985, 75, 443-455.

(7) Stokowski, L. A. Fundamentals of Phototherapy for Neonatal Jaundice. Adv. Neonatal Care 2011, 11, 10-21.

(8) Wang, J.; Guo, G. X.; Li, A. M.; Cai, W. Q.; Wang, X. W. Challenges of Phototherapy for Neonatal Hyperbilirubinemia (Review). Exp. Ther. Med. 2021, 21, 231.

(9) Vitek, L. Bilirubin as a Signaling Molecule. Med. Res. Rev. 2020, 40, 1335-1351.

(10) Zietz, B.; Gillbro, T. Initial Photochemistry of Bilirubin Probed by Femtosecond Spectroscopy. J. Phys. Chem. B 2007, 111, 1199712003.

(11) Zietz, B.; Blomgren, F. Conical Intersection in a Bilirubin Model - a Possible Pathway for Phototherapy of Neonatal Jaundice. Chem. Phys. Lett. 2006, 420, 556-561.

(12) Zietz, B.; Macphersona, A. N.; Gillbro, T. Resolution of Ultrafast Excited State Kinetics of Bilirubin in Chloroform and Bound to Human Serum Albumin. Phys. Chem. Chem. Phys. 2004, 6, 45354537.

(13) Mazzoni, M.; Agati, G.; Troup, G.; Pratesi, R. Analysis of Wavelength-Dependent Photoisomerization Quantum Yields in Bilirubins by Fitting Two Exciton Absorption Bands. J. Opt. A: Pure Appl. Opt. 2003, 5, No. S374.

(14) Sloper, R.; Truscott, T. Excited States of Bilirubin. Photochem. Photobiol. 1980, 31, 445-450.

(15) Petersen, C. E.; Ha, C. E.; Harohalli, K.; Feix, J. B.; Bhagavan, N. V. A Dynamic Model for Bilirubin Binding to Human Serum Albumin. J. Bio. Chem. 2000, 275, 20985-20995.

(16) Sato, H.; Honore, B.; Brodersen, R. Multiple Binding of Bilirubin to Human-Serum Albumin and Cobinding with Laurate. Arch. Biochem. Biophys. 1988, 260, 811-821.

(17) Ziberna, L.; Martelanc, M.; Franko, M.; Passamonti, S. Bilirubin Is an Endogenous Antioxidant in Human Vascular Endothelial Cells. Sci. Rep. 2016, 6, 29240.

(18) Weisiger, R. A.; Ostrow, J. D.; Koehler, R. K.; Webster, C. C.; Mukerjee, P.; Pascolo, L.; Tiribelli, C. Affinity of Human Serum Albumin for Bilirubin Varies with Albumin Concentration and Buffer Composition - Results of a Novel Ultrafiltration Method. J. Biol. Chem. 2001, 276, 29953-29960.

(19) Madea, D.; Mahvidi, S.; Chalupa, D.; Mujawar, T.; Dvorak, A.; Muchova, L.; Janos, J.; Slavicek, P.; Svenda, J.; Vitek, L.; et al. Wavelength-Dependent Photochemistry and Biological Relevance of a Bilirubin Dipyrrinone Subunit. J. Org. Chem. 2020, 85, 13015-13028.

(20) Lamola, A. A., Optical Properties and Structure of Tetrapyrroles. De Gruyter: Berlin, 1985.

(21) El-Zohry, A. M.; Orthaber, A.; Zietz, B. Isomerization and Aggregation of the Solar Cell Dye D149. J. Phys. Chem. C 2012, 116, 26144-26153.

(22) Chai, J.-D.; Head-Gordon, M. Long-Range Corrected Hybrid Density Functionals with Damped Atom-Atom Dispersion Corrections. 2008, 10 (44), 6615-6620, DOI: 10.1039/B810189B.

(23) Bonnett, R.; Davies, J. E.; Hursthouse, M. B.; Sheldrick, G. M. The Structure of Bilirubin. Proc. R. Soc. London, Ser. B 1978, 202, 249-268.
(24) Tomasi, J.; Mennucci, B.; Cammi, R. Quantum Mechanical Continuum Solvation Models. 2005, 105 (8), 2999-3094, DOI: $10.1021 /$ cr9904009.

(25) Plasser, F. Theodore: A Toolbox for a Detailed and Automated Analysis of Electronic Excited State Computations. J. Chem. Phys. 2020, 152, No. 084108.

(26) Martin, R. L. J. Natural Transition Orbitals. J. Chem. Phys. 2003, 118, 4775-4777.

(27) Frisch, M. J.; Trucks, G. W.; Schlegl, H. B.; Scuseria, G. E.; Robb, M. A.; Cheeseman, J. R.; Scalmani, G.; Barone, V.; Mennucci, B.; Petersson, G. A., et al. Gaussian 16, Rev. A03, Gaussian Inc.: 2016.

(28) Rohmer, T.; Matysik, J.; Mark, F. Solvation and Crystal Effects in Bilirubin Studied by Nmr Spectroscopy and Density Functional Theory. J. Phys. Chem. A 2011, 115, 11696-11714.

(29) El-Zohry, A. M.; Alturki, A.; Yin, J.; Mallick, A.; Shekhah, O.; Eddaoudi, M.; Ooi, B. S.; Mohammed, O. F. Tunable Twisting Motion of Organic Linkers Via Concentration and Hydrogen-Bond Formation. J. Phys. Chem. C 2019, 123, 5900-5906.

(30) El-Zohry, A. M.; Roca-Sanjuan, D.; Zietz, B. Ultrafast Twisting of the Indoline Donor Unit Utilized in Solar Cell Dyes: Experimental and Theoretical Studies. J. Phys. Chem. C 2015, 119, 2249-2259.

(31) Yang, C.; El-Demellawi, J. K.; Yin, J.; Velusamy, D. B.; Emwas, A.-H. M.; El-Zohry, A. M.; Gereige, I.; AlSaggaf, A.; Bakr, O. M.; Alshareef, H. N. Mapbi3 Single Crystals Free from Hole-Trapping Centers for Enhanced Photodetectivity. ACS Energy Lett. 2019, 4, 2579-2584.

(32) El-Zohry, A. M.; Shaheen, B. S.; Burlakov, V. M.; Yin, J.; Hedhili, M. N.; Shikin, S.; Ooi, B.; Bakr, O. M.; Mohammed, O. F. Extraordinary Carrier Diffusion on Cdte Surfaces Uncovered by $4 \mathrm{~d}$ Electron Microscopy. Chem 2019, 5, 497-499.

(33) Shaheen, B. S.; El-Zohry, A. M.; Zhao, J.; Yin, J.; Hedhili, M. N.; Bakr, O. M.; Mohammed, O. F. Real-Space Mapping of SurfaceOxygen Defect States in Photovoltaic Materials Using Low-Voltage Scanning Ultrafast Electron Microscopy. ACS Appl. Mater. Interfaces 2020, 12, 7760-7767.

(34) Lakowicz, J. R., Principles of Fluorescence Spectroscopy. Springer, 2007.

(35) Moglich, A.; Joder, K.; Kiefhaber, T. End-to-End Distance Distributions and Intrachain Diffusion Constants in Unfolded Polypeptide Chains Indicate Intramolecular Hydrogen Bond Formation. Proc. Natl. Acad. Sci. U. S. A. 2006, 103, 12394-12399.

(36) Liu, D.; El-Zohry, A. M.; Taddei, M.; Matt, C.; Bussotti, L.; Wang, Z.; Zhao, J.; Mohammed, O. F.; Di Donato, M.; Weber, S. Long-Lived Charge-Transfer State Induced by Spin-Orbit Charge Transfer Intersystem Crossing (Soct-Isc) in a Compact Spiro Electron Donor/Acceptor Dyad. Angew. Chem., Int. Ed. Engl. 2020, 59, 1159111599.

(37) Imran, M.; El-Zohry, A. M.; Matt, C.; Taddei, M.; Doria, S.; Bussotti, L.; Foggi, P.; Zhao, J.; Di Donato, M.; Mohammed, O. F.; et al. Intersystem Crossing Via Charge Recombination in a PeryleneNaphthalimide Compact Electron Donor/Acceptor Dyad. J. Mater. Chem. C 2020, 8, 8305-8319.

(38) Hussain, M.; El-Zohry, A. M.; Gobeze, H. B.; Zhao, J. Z.; D'Souza, F.; Mohammed, O. F. Intramolecular Energy and Electron Transfers in Bodipy Naphthalenediimide Triads. J. Phys. Chem. A 2018, 122, 6081-6088.

(39) Lamola, A. A.; Flores, J. Effect of Buffer Viscosity on the Fluorescence of Bilirubin Bound to Human Serum Albumin. J. Am. Chem. Soc. 1982, 104, 2530-2534.

(40) El-Zohry, A. M.; Zietz, B. Concentration and Solvent Effects on the Excited State Dynamics of the Solar Cell Dye D149: The Special Role of Protons. J. Phys. Chem. C 2013, 117, 6544-6553.

(41) El-Zohry, A. M. The Origin of Slow Electron Injection Rates for Indoline Dyes Used in Dye-Sensitized Solar Cells. Dyes Pigm. 2019, 160, 671-674.

(42) El-Zohry, A. M.; Karlsson, M. Gigantic Relevance of Twisted Intramolecular Charge Transfer for Organic Dyes Used in Solar Cells. J. Phys. Chem. C 2018, 122, 23998-24003. 
(43) Abdelhamid, H. N.; El-Zohry, A. M.; Cong, J.; Thersleff, T.; Karlsson, M.; Kloo, L.; Zou, X. Towards Implementing Hierarchical Porous Zeolitic Imidazolate Frameworks in Dye-Sensitized Solar Cells. R. Soc. Open Sci. 2019, 6, No. 190723.

(44) Sirbu, D.; Karlsson, J. K. G.; Harriman, A. Nonradiative Decay Channels for a Structurally-Distorted, Monostrapped Bodipy Derivative. J. Phys. Chem. A 2018, 122, 9160-9170.

(45) Shirakov, A.; Burshtein, Z.; Katzir, A.; Frumker, E.; Ishaaya, A. A. Competing Radiative and Nonradiative Decay of Embedded Ions States in Dielectric Crystals: Theory, and Application to $\mathrm{Co}^{2+}: \mathrm{Agcl}_{0.5} \mathrm{br}_{0.5}$. Opt. Express 2018, 26, 11694-11707.

(46) El-Zohry, A. M.; Agrawal, S.; De Angelis, F.; Pastore, M.; Zietz, B. Critical Role of Protons for Emission Quenching of Indoline Dyes in Solution and on Semiconductor Surfaces. J. Phys. Chem. C 2020, 124, 21346-21356.

(47) Itoh, S.; Okada, H.; Kuboi, T.; Kusaka, T. Phototherapy for Neonatal Hyperbilirubinemia. Pediatr Int 2017, 59, 959-966.

(48) Ebbesen, F.; Madsen, P. H.; Vandborg, P. K.; Jakobsen, L. H.; Trydal, T.; Vreman, H. J. Bilirubin Isomer Distribution in Jaundiced Neonates During Phototherapy with Led Light Centered at $497 \mathrm{Nm}$ (Turquoise) Vs. 459 Nm (Blue). Pediatr. Res. 2016, 80, 511-515.

(49) Brewster, D. H.; Tucker, J. S.; Fleming, M.; Morris, C.; Stockton, D. L.; Lloyd, D. J.; Bhattacharya, S.; Chalmers, J. W. Risk of Skin Cancer after Neonatal Phototherapy: Retrospective Cohort Study. Arch. Dis. Child. 2010, 95, 826-831. 